\title{
TWO-TERM PARTIAL TILTING COMPLEXES OVER BRAUER TREE ALGEBRAS
}

\author{
Mikhail Antipov and Alexandra Zvonareva
}

\begin{abstract}
In this paper we describe all indecomposable two-term partial tilting complexes over a Brauer tree algebra with multiplicity 1 using a criterion for a minimal projective presentation of a module to be a partial tilting complex. As an application we describe all two-term tilting complexes over Brauer star algebra and compute their endomorphism rings.
\end{abstract}

\section{Introduction}

In [1] Rouquier and Zimmermann defined a derived Picard group $\operatorname{Tr} \operatorname{Pic}(A)$ of an algebra $A$, i. e. a group of autoequivalences of the derived category of $A$, given by multiplication by a two-sided tilting complex modulo natural isomorphism. The tensor product of two-sided tilting complexes gives the multiplication in this group. Despite the fact that for a Brauer tree algebra with the multiplicity of the exceptional vertex 1 several braid group actions on $\operatorname{Tr} \operatorname{Pic}(A)$ are known ([1], 2]), the whole derived Picard group is computed only for an algebra with two simple modules ([1]).

On the other hand Abe and Hoshino showed that over a selfinjective artin algebra of finite representation type any tilting complex $P$ such that $\operatorname{add}(P)=\operatorname{add}(\nu P)$, where $\nu$ is the Nakayama functor, can be presented as a product of tilting complexes of length $\leq 1$ (3]). Therefore instead of considering the derived Picard group we can consider the derived Picard groupoid corresponding to some class of derived equivalent algebras. The objects of this groupoid are the algebras from this class and the morphisms are the derived equivalences given by multiplication by a two-sided tilting complex modulo natural isomorphism. For example, one can consider the derived Picard groupoid corresponding to the class of Brauer tree algebras with fixed number of simple modules and multiplicity $k$ (the algebras from this class are derived equivalent and this class is closed under derived equivalence). Then the result of Abe and Hoshino means that the derived Picard groupoid corresponding to the class of Brauer tree algebras with fixed number of simple modules and multiplicity $k$ is generated by one-term and two-term tilting complexes. 
In this paper we give a criterion for a minimal projective presentation of a module without projective direct summands to be a partial tilting complex, namely we have the following:

Proposition 1 Let $A$ be a selfinjective $K$-algebra, $M$ be a module without projective direct summands and let $T:=P^{0} \stackrel{f}{\rightarrow} P^{1}$ be a minimal projective presentation of module $M$. Complex $T$ is partial tilting if and only if $\operatorname{Hom}_{A}\left(M, \Omega^{2} M\right)=0$ and $\operatorname{Hom}_{K^{b}(A)}(T, M)=0$.

In Proposition 1 module $M$ is considered as a stalk complex concentrated in degree 0 , complex $T:=P^{0} \stackrel{f}{\rightarrow} P^{1}$ is concentrated in degrees 0 and 1 accordingly.

Using this proposition we classify all indecomposable two-term partial tilting complexes over a Brauer tree algebra with multiplicity 1.

Theorem 1 Let $A$ be a Brauer tree algebra with multiplicity 1. A minimal projective presentation of an indecomposable non-projective $A$-module $M$ is a partial tilting complex if and only if $M$ is not isomorphic to $P / \operatorname{soc}(P)$ for any indecomposable projective module $P$.

Hopefully it will allow us to obtain a full classification of two-term tilting complexes over Brauer tree algebras. As an illustration we describe all two-term tilting complexes over Brauer star algebra and compute their endomorphism rings (for an arbitrary multiplicity) in sections 5 and 6 . Note that the results in sections 5 and 6 partially intersect with [4, [5].

Acknowledgement: We would like to thank Alexander Generalov for his helpful remarks.

\section{Preliminaries}

Let $K$ be an algebraically closed field, $A$ be a finite dimensional algebra over $K$. We will denote by $A$-mod the category of finitely generated left $A$-modules, by $K^{b}(A)$ - the bounded homotopy category and by $D^{b}(A)$ the bounded derived category of $A$-mod. The shift functor on the derived category will be denoted by [1]. Let us denote by $A$-perf the full subcategory of $D^{b}(A)$ consisting of perfect complexes, i.e. of bounded complexes of finitely generated projective $A$-modules. In the path algebra of a quiver the product of arrows $\stackrel{a}{\rightarrow} \stackrel{b}{\rightarrow}$ will be denoted by $a b$. For convenience all algebras are supposed to be basic.

Definition 1. A complex $T \in A$-perf is called tilting if

1) $\operatorname{Hom}_{D^{b}(A)}(T, T[i])=0$, for $i \neq 0$;

2) $T$ generates $A$-perf as a triangulated category.

Tilting complexes were defined by Rickard ([6]) and play an essential role in the study of the equivalences of derived categories.

Definition 2. A complex $T \in A$-perf is called partial tilting if the condition 1 from definition 1 is satisfied. 
Definition 3. A tilting complex $T \in A$-perf is called basic if it does not contain isomorphic direct summands or equally if $\operatorname{End}_{D^{b}(A)}(T)$ is a basic algebra.

We will call a (partial) tilting complex a two-term (partial) tilting complex if it is concentrated in two neighboring degrees.

Definition 4. An algebra $A$ is called special biserial (SB-algebra), if $A$ is isomorphic to $K Q / I$ for some quiver $Q$ and an admissible ideal of relations $I$, and the following is satisfied:

1) any vertex of $Q$ is the starting point of at most two arrows;

2) any vertex of $Q$ is the end point of at most two arrows;

3) if $b$ is an arrow in $Q$ then there is at most one arrow a such that $a b \notin I$;

4) if $b$ is an arrow in $Q$ then there is at most one arrow $c$ such that $b c \notin I$.

For an SB-algebra the full classification of indecomposable modules up to isomorphism is known ([7], [8]).

Definition 5. Let $B$ be a symmetric SB-algebra over a field $K$. A-cycle is a maximal ordered set of nonrepeating arrows of $Q$ such that the product of any two neighboring arrows is not equal to zero.

Note that the fact that algebra is symmetric means that $A$-cycles are actually cycles. Also sometimes just a maximal ordered set of arrows of $Q$ such that the product of any two neighboring arrows is not equal to zero is called an $A$-cycle (see 9]). Note also that in this case $A$-cycles are maximal nonzero paths in $B$.

An important example of an SB-algebra of finite representation type is a Brauer tree algebra. Also these algebras play an important role in modular representation theory of finite groups.

Definition 6. Let $\Gamma$ be a tree with $n$ edges and an exceptional vertex which has an assigned multiplicity $k \in \mathbb{N}$. Let us fix a cyclic ordering of the edges adjacent to each vertex in $\Gamma$ (if $\Gamma$ is embedded into plane we will assume that the cyclic ordering is clockwise). In this case $\Gamma$ is called a Brauer tree of type $(n, k)$.

For a Brauer tree of type $(n, k)$ one can associate a finite dimensional algebra $A(n, k)$. Algebra $A(n, k)$ is an algebra with $n$ simple modules $S_{i}$ which are in one to one correspondence with edges $i \in \Gamma$. The two series of composition factors of an indecomposable projective module $P_{i}$ (with top $S_{i}$ ) are obtained by going anticlockwise around the $i$-th vertex. We go around the $i$-th vertex $k$ times if the vertex is exceptional and one time if it is not. The full description of the Brauer tree algebras in terms of composition factors is given in [10].

Furthermore, Rickard showed that two Brauer tree algebras corresponding to the trees $\Gamma$ and $\Gamma^{\prime}$ are derived equivalent if and only if their types $(n, k)$ and $\left(n^{\prime}, k^{\prime}\right)$ coincide (11]) and it follows from the results of Gabriel and Riedtmann that this class is closed under derived equivalence ([12]). 


\section{Two-term tilting complexes over selfinjective algebras}

Let $A$ be an arbitrary finite dimensional selfinjective $K$-algebra.

Lemma 1. Any two-term complex $T:=P^{0} \stackrel{f}{\rightarrow} P^{1} \in A$-perf is isomorphic to a direct sum of the minimal projective presentation of a module and a stalk complex of projective module concentrated in degree 0.

Proof Let us denote by $M$ the cokernel of $f$. The minimal projective presentation of $M$ is a direct summand of $T$. So $T$ is a direct sum of the minimal projective presentation of $M$, some stalk complex $P^{0}$ concentrated in degree 0 , which can be zero and on which $f$ acts as a zero map, and a complex of the form $P \stackrel{\text { id }}{\rightarrow} P$, which is homotopic to 0 .

We will suppose that the minimal projective presentation of a module is concentrated in degrees 0 and 1 in cohomological notation. For the sake of simplicity we will consider only minimal projective presentations of modules without projective summands. Direct summands corresponding to stalk complexes of projective modules concentrated in degree 1 will be considered separately in Proposition 2.

Proposition 1. Let $A$ be a selfinjective $K$-algebra, $M$ be a module without projective direct summands and let $T:=P^{0} \stackrel{f}{\rightarrow} P^{1}$ be a minimal projective presentation of module $M$. Complex $T$ is partial tilting if and only if $\operatorname{Hom}_{A}\left(M, \Omega^{2} M\right)=0$ and $\operatorname{Hom}_{K^{b}(A)}(T, M)=0$.

Proof Let $h: P^{1} \rightarrow P^{0}$ be such that $h f=0=f h$, i.e. $h$ gives a morphism $T \rightarrow T[-1]$.

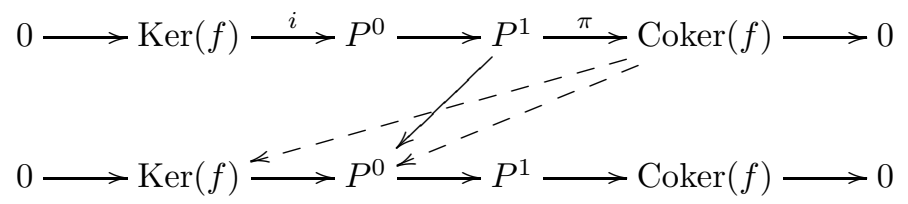

The condition $h f=0$ means that $\operatorname{Im}(f) \subseteq \operatorname{Ker}(h)$, consequently $h$ goes through $\operatorname{Coker}(f)$, i.e. there exists $h^{\prime} \in \operatorname{Hom}_{A}\left(\operatorname{Coker}(f), P^{0}\right)$ such that $h=h^{\prime} \pi$, but $\pi$ is surjective, hence $\operatorname{Im}(h)=\operatorname{Im}\left(h^{\prime}\right)$.

The condition $f h=0$ means that $\operatorname{Im}\left(h^{\prime}\right)=\operatorname{Im}(h) \subseteq \operatorname{Ker}(f)$ consequently $h^{\prime}$ goes through $\operatorname{Ker}(f)$, i.e. there exists $h^{\prime \prime}$ such that $h^{\prime}=i h^{\prime \prime}, h=i h^{\prime \prime} \pi$. Note that since $\pi$ is surjective and $i$ is injective, $h=0$ if and only if $h^{\prime \prime}=0$.

Also if there is a nonzero $h^{\prime \prime} \in \operatorname{Hom}_{A}(\operatorname{Coker}(f), \operatorname{Ker}(f))$ a morphism $h=$ $i h^{\prime \prime} \pi$ gives a nonzero morphism $T \rightarrow T[-1]$. So

$$
\operatorname{Hom}_{D^{b}(A)}(T, T[-1])=0 \Leftrightarrow \operatorname{Hom}_{A}\left(M, \Omega^{2} M\right)=0 .
$$

Let us now verify that

$$
\operatorname{Hom}_{D^{b}(A)}(T, T[1])=0 \Leftrightarrow \operatorname{Hom}_{K^{b}(A)}(T, M)=0 .
$$


We have that $\operatorname{Hom}_{D^{b}(A)}(T, T[1])=\operatorname{Hom}_{D^{b}(A)}(T, P \bullet)=\operatorname{Hom}_{D^{b}(A)}(T, M)$, where $P_{\bullet}$ is the projective resolution of $M$. Since $T$ consists of projective modules, $\operatorname{Hom}_{D^{b}(A)}(T, M)=\operatorname{Hom}_{K^{b}(A)}(T, M)$.

Corrolary 1. The projective presentation of a band-module over a symmetric SB-algebra can not be a partial tilting complex.

Proof In the Auslander-Reiten quiver all band-modules lie on 1-tubes ([13]), so $\Omega^{2} M=M$.

The proof of the next statement is analogous to the proof of Proposition 1.

Proposition 2. Let $A$ be a selfinjective $K$-algebra, $M$ be a module without projective direct summands such that its minimal projective presentation is a partial tilting complex.

The sum of a stalk complex of projective module $P$ concentrated in degree 0 and the minimal projective presentation of module $M$ is a partial tilting complex if and only if $\operatorname{Hom}_{A}(M, P)=0=\operatorname{Hom}_{A}(P, M)$.

The sum of a stalk complex of projective module $P$ concentrated in degree 1 and the minimal projective presentation of module $M$ is a partial tilting complex if and only if $\operatorname{Hom}_{A}\left(\Omega^{2} M, P\right)=0=\operatorname{Hom}_{A}\left(P, \Omega^{2} M\right)$.

\section{Two-term tilting complexes over Brauer tree algebras with multiplicity 1}

The next remark (14]) plays an important role.

Remark 1. Let $A$ be a finite dimensional algebra over a field $K$, let proj- $A$ and inj- $A$ be the categories of finitely generated projective and injective modules respectively, $K^{b}$ (proj- $A$ ), $K^{b}$ (inj- $A$ ) bounded homotopy categories, $D$ the duality of the module category with respect to $K$. Then the Nakayama functor $\nu$ induces an equivalence of triangulated categories $K^{b}(\operatorname{proj}-A) \rightarrow K^{b}(\mathrm{inj}-A)$ and there is a natural isomorphism $\operatorname{DHom}(P,-) \rightarrow \operatorname{Hom}(-, \nu P)$ for $P \in K^{b}(\operatorname{proj}-A)$.

In the case of the symmetric algebra it means that for $T \in A$-perf the condition $\operatorname{Hom}_{D^{b}(A)}(T, T[1])=0$ is satisfied if and only if $\operatorname{Hom}_{D^{b}(A)}(T, T[-1])=$ 0 .

From now on in this section we will consider only Brauer tree algebras $A$ corresponding to a Brauer tree $\Gamma$ such that the multiplicity of the exceptional vertex of $\Gamma$ is 1 . Let us fix an $A$-module $M$ and let us denote by $T:=P^{0} \stackrel{f}{\rightarrow} P^{1}$ its minimal projective presentation.

Lemma 2. Let $M$ be an indecomposable nonprojective $A$-module. The condition $\operatorname{Hom}_{A}\left(P^{0}, M\right)=0$ implies $\operatorname{Hom}_{A}\left(M, \Omega^{2} M\right)=0$ and $\operatorname{Hom}_{K^{b}(A)}(T, M)=0$.

Proof The condition $\operatorname{Hom}_{A}\left(P^{0}, M\right)=0$ obviously implies $\operatorname{Hom}_{K^{b}(A)}(T, M)=0$. 
Let us show that $\operatorname{Hom}_{A}\left(P^{0}, M\right)=0$ implies $\operatorname{Hom}_{A}\left(M, \Omega^{2} M\right)=0$. Since $\operatorname{Hom}_{A}\left(P^{0}, M\right)=0$, there is no composition factor in $M$ isomorphic to a direct summand of $\operatorname{top}\left(P^{0}\right)=\operatorname{soc}\left(P^{0}\right)$. The module $\Omega^{2} M$ is a submodule of $P^{0}$, hence $\operatorname{soc}\left(\Omega^{2} M\right) \subseteq \operatorname{soc}\left(P^{0}\right)$. For any $h \in \operatorname{Hom}_{A}\left(M, \Omega^{2} M\right)$ we have that $\operatorname{Im}(h) \cap$ $\operatorname{soc}\left(\Omega^{2} M\right)=0$, hence $h=0$.

Lemma 3. Let $M$ be a nonprojective A-module such that $\operatorname{dim}(\operatorname{top}(M))=1$. The minimal projective presentation of $M$ is a partial tilting complex if and only if $M$ is not isomorphic to $P / \operatorname{soc}(P)$ for any indecomposable projective module $P$.

Proof The condition $\operatorname{dim}(\operatorname{top}(M))=1$ implies that $M \simeq P^{1} / U$, where $P^{1}$ is indecomposable.

If $U=\operatorname{soc}\left(P^{1}\right)$, then $P^{0} \simeq P^{1}$ because $A$ is symmetric. Hance $\Omega^{2} M$ is a submodule of $P^{1}$, hence, $\operatorname{soc}\left(\Omega^{2} M\right)=\operatorname{soc}\left(P^{1}\right)=\operatorname{top}\left(P^{1}\right)=\operatorname{top}(M)$, which

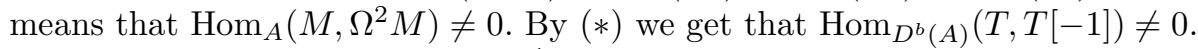

Let us assume that $U \neq \operatorname{soc}\left(P^{1}\right)$. We denote by $I$ the set of indexes corresponding to composition factors of $\operatorname{top}(U)$. The projective cover of $U$ is isomorphic to $\bigoplus_{i \in I} A e_{i}$. Since $U \neq \operatorname{soc}\left(P^{1}\right)$, the set $I$ does not contain the indexes corresponding to $\operatorname{soc}\left(P^{1}\right)$ or to composition factors of $P^{1} / U$ (over a Brauer tree algebra with multiplicity 1 all composition factors of an indecomposable projective module except for the top and the socle are distinct). Hance $\operatorname{Hom}_{A}\left(P^{0}, M\right)=0$. By Lemma 2 and Proposition 1 the minimal projective presentation of $P^{1} / U$ is a partial tilting complex.

Let us denote by $C F(L)$ the set of the composition factors of module $L$.

Lemma 4. For any indecomposable nonprojective A-module $M$ such that $\operatorname{dim}(\operatorname{top}(M)) \geq 2$ the condition $\operatorname{Hom}_{K^{b}(A)}(T, M)=0$ is satisfied.

Proof Note that $\operatorname{dim}(\operatorname{top}(M)) \geq 2$ implies $C F\left(\operatorname{top}\left(P^{0}\right)\right) \cap C F(M) \subseteq$ $\operatorname{soc}(M)$. Indeed, since over a Brauer tree algebra with multiplicity 1 all composition factors of an indecomposable nonprojective module are distinct, $C F\left(\operatorname{top}\left(P^{0}\right)\right) \cap C F(M) \subseteq \operatorname{soc}(M)$. Consequently, for any morphism $h: P^{0} \rightarrow M$ the following holds $\operatorname{Im}(h) \subseteq \operatorname{soc}(M)$, hence Ker $h \supseteq \operatorname{rad}\left(P^{0}\right) \supseteq \operatorname{Ker} f$, hence $h$ goes through $f$ and $h=0$ in $K^{b}(A)$.

Finally we have:

Theorem 1. A minimal projective presentation of an indecomposable nonprojective A-module $M$ is a partial tilting complex if and only if $M$ is not isomorphic to $P / \operatorname{soc}(P)$ for any indecomposable projective module $P$.

Proof The case $\operatorname{dim}(\operatorname{top}(M))=1$ is dealt with in Lemma 3 ; in the case $\operatorname{dim}(\operatorname{top}(M)) \geq 2$ the required result holds because of Lemma 4 Remark 1 and (**). 


\section{Two-term tilting complexes over Brauer star algebra}

Let us consider a quiver $Q$ :

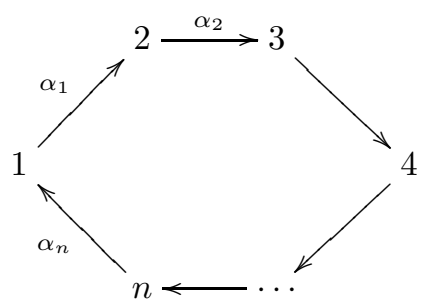

The vertices of the quiver are numbered by elements of $\mathbb{Z} / n \mathbb{Z}$. Consider the ideal $I$ generated by relations

$$
I:=\left\langle\left(\alpha_{i} \cdot \alpha_{i+1} \cdot \ldots \cdot \alpha_{i-1}\right)^{k} \cdot \alpha_{i}, i=1, \ldots, n\right\rangle .
$$

Set $A=k Q / I$. We denote by $e_{i}$ the path of length 0 corresponding to the vertex $i$.

Any indecomposable module over this algebra is uniserial, in particular any indecomposable module is uniquely determined by the ordered set of its composition factors. We will denote a module by the set of the indexes corresponding to its composition factors ordered from the top to the socle. For example, the simple module corresponding to the idempotent $e_{i}$ will be denoted by $(i)$.

In the previous section the description of all two-term partial tilting complexes in the case $k=1$ was given. Now we will describe such complexes over a Brauer star algebra for an arbitrary $k$.

Proposition 3. The minimal projective presentation of an indecomposable Amodule is a partial tilting complex if and only if $l(M)<n$, where $l(M)$ is the length of $M$.

Proof If $|C F(M)|>n-1$ then both $M$ and $\Omega^{2} M$ contain all simple modules as composition factors. In particular, $\operatorname{top}(M)$ is a composition factor of $\Omega^{2} M$ hence $\operatorname{Hom}_{A}\left(M, \Omega^{2} M\right) \neq 0$. If $|C F(M)|<n$, then in $\Omega^{2} M$ there is no composition factor isomorphic to $\operatorname{top}(M)$ hence $\operatorname{Hom}_{A}\left(M, \Omega^{2} M\right)=0$. It is also clear that $\operatorname{Hom}_{K^{b}(A)}(T, M)=0$, since there is no composition factor isomorphic to top $\left(P^{0}\right)$ in $M$.

Let us describe all two-term tilting complexes over $A$, concentrated in degrees 0 and 1 . Let there be given two modules $M=(i, i-1, \ldots, j)$ and $N=(m, m-$ $1, \ldots, l)$ such that the number of composition factors of $M$ and of $N$ is less then $n$. Let $T$ be the minimal projective presentation of $M, T^{\prime}$ be the minimal projective presentation of $N$. Note that $\Omega^{2} M=(i-1, \ldots, j-1), \Omega^{2} N=(m-1, \ldots, l-1)$. Let us state when the sum of the minimal projective presentations of $M$ and $N$ is a partial tilting complex. 
$\operatorname{Hom}_{A}\left(M, \Omega^{2} N\right)=0$ if and only if $i \notin\{m-1, m-2, \ldots, l-1\}$ or $i \in$ $\{m-1, m-2, \ldots, l-1\}$, but $j \in\{i, i-1, \ldots, l\}$.

$\operatorname{Hom}_{A}\left(N, \Omega^{2} M\right)=0$ if and only if $m \notin\{i-1, i-2, \ldots, j-1\}$ or $m \in$ $\{i-1, i-2, \ldots, j-1\}$, but $l \in\{m, m-1, \ldots, j\}$.

Analysing these conditions we conclude that either the sets $\{i, i-1, \ldots, j-1\}$, $\{m, m-1, \ldots, l-1\}$ do not intersect or one lies inside the other.

Now let us figure out when a sum of the minimal projective presentation of a module $M=(i, i-1, \ldots, j)$ and a stalk complex of a projective module $P_{m}=(m, m-1, \ldots, m)$ concentrated in degree 0 is a partial tilting complex.

$\operatorname{Hom}_{A}(M, P)=0=\operatorname{Hom}_{A}(P, M)$ if and only if $m \notin\{i, i-1, \ldots, j\}$.

Similarly, a sum of the minimal projective presentation of a module $M=$ $(i, i-1, \ldots, j)$ and a stalk complex of a projective module $P_{m}=(m, m-$ $1, \ldots, m)$ concentrated in degree 1 is a partial tilting complex if and only if $\operatorname{Hom}_{A}\left(\Omega^{2} M, P\right)=0=\operatorname{Hom}_{A}\left(P, \Omega^{2} M\right)$, i.e. $m \notin\{i-1, i-2, \ldots j-1\}$.

Note also that all stalk complexes of projective modules are concentrated either in degree 0 or in degree 1 , since for any two projective modules $P_{m}, P_{l}$ over a Brauer star algebra $\operatorname{Hom}_{A}\left(P_{m}, P_{l}\right) \neq 0$.

It is known that in the case of a symmetric algebra of finite representation type any partial tilting complex with $n$ (where $n$ is the number of isoclasses of simple modules) nonisomorphic direct summands is tilting ([3]). Thus to describe all two-term tilting complexes is the same as to describe all configurations of $n$ pairwise orthogonal indecomposable complexes, each of which is either a minimal projective presentation of an indecomposable module $M$ such that the number of composition factors of $M$ is less then $n$ or a stalk complex of a projective module concentrated in degree 0 or degree 1 , i.e. of $n$ complexes which pairwisely satisfy the conditions stated before.

We will call an interval a set of vertices of an $n$-gon taken in order with marked starting point and end point. The covering $S$ of an $n$-gon by distinguished intervals is the following structure: an $n$-gon with a partition of its vertices into noncrossing intervals (we call them outer), each interval can contain from 1 to $n$ vertices; in each outer interval containing $r(r>1)$ vertices $r-2$ inner intervals are additionally chosen, each of which contains more that 1 vertex; inner intervals either do not intersect or lie one inside the other. Also in each outer interval $(i, i-1, \ldots, j)$ with length greater than 1 we will pick out an interval of length 1 as follows: it is either a starting point for all outer intervals or an end point. Note that the covering contains exactly $n$ intervals. To such a covering $S$ one can assign a two-term tilting complex $T_{S}$ as follows.

We will consider two cases:

1) To all outer intervals $(i, i-1, \ldots, j) \in S$ of length greater than 1 an inner interval $(j)$ of length 1 is assigned. Let us construct a tilting complex as follows: for each interval $(i, i-1, \ldots, j)$ containing more than 1 vertex take a module $M=(i, i-1, \ldots, j+1)$, as a direct summand of the tilting complex take the minimal projective presentation of $M$. For each interval containing 1 vertex take a stalk complex of the projective module corresponding to this vertex concentrated in degree 0 . In this way we get $n$ summands.

2) To all outer intervals $(i, i-1, \ldots, j) \in S$ of length greater than 1 an inner 
interval $(i)$ of length 1 is assigned. As before for each interval $(i, i-1, \ldots, j)$ containing more than 1 vertex take a module $M=(i, i-1, \ldots, j+1)$, as a direct summand of the tilting complex take the minimal projective presentation of $M$. For each interval containing 1 vertex take a stalk complex of the projective module corresponding to this vertex concentrated in degree 1 . In this way we get $n$ summands.

To the trivial covering, containing only intervals of length 1 , two tilting complexes: $A$ and $A[-1]$ are assigned.

Based on the previous construction we get the following:

Proposition 4. Over a Brauer star algebra with $n$ vertices and multiplicity $k$ the set of all basic two-term tilting complexes not isomorphic to $A$ or $A[-1]$ is in one to one correspondence with the set of all nontrivial coverings of an n-gon by distinguished intervals.

\section{Endomorphism rings}

Let us construct the endomorphism ring of a two-term tilting complex over a Brauer star algebra with $n$ vertices and multiplicity $k$, i.e. the endomorphism rings of a tilting complex corresponding to the covering $S$ of an $n$-gon. It is well known that it is isomorphic to a Brauer tree algebra corresponding to some Brauer tree $\Gamma$ with multiplicity $k$. For this purpose we first compute the Cartan matrix of the algebra $\operatorname{End}_{K^{b}(A)}\left(T_{S}\right)$. It will tell us which edges of $\Gamma$ are incident to one vertex. After that we will only have to establish the cyclic ordering of the edges incident to each vertex of $\Gamma$. It is easy to compute the Cartan matrix of $\operatorname{End}_{K^{b}(A)}\left(T_{S}\right)$ using the well known formulae by Happel [15]: let $Q=\left(Q^{r}\right)_{r \in \mathbb{Z}}, R=\left(R^{s}\right)_{s \in \mathbb{Z}} \in A$-perf, then

$$
\sum_{i}(-1)^{i} \operatorname{dim}_{K} \operatorname{Hom}_{K^{b}(A)}(Q, R[i])=\sum_{r, s}(-1)^{r-s} \operatorname{dim}_{K} \operatorname{Hom}_{A}\left(Q^{r}, R^{s}\right) .
$$

Note that if $\operatorname{Hom}_{K^{b}(A)}(Q, R[i])=0, i \neq 0$ (for example, in the case when $Q$ and $R$ are summands of a tilting complex) then the left hand side of the formulae becomes $\operatorname{dim}_{K} \operatorname{Hom}_{K^{b}(A)}(Q, R)$.

As before we will consider two cases:

1) To all outer intervals $(i, i-1, \ldots, j) \in S$ of length greater than 1 an inner interval $(j)$ of length 1 is assigned, i.e. all stalk complexes of projective modules which are direct summands of $T_{S}$ are concentrated in degree 0 . Let $(i, i-1, \ldots, j)$, $(t, t-1, \ldots, l) \in S$ be two arbitrary intervals of the covering $S$ of length greater then 1 . And let $(m),(r) \in S$ be intervals of length 1 . It is easy to see that

$$
\begin{gathered}
\operatorname{dim}\left(\operatorname{Hom}_{K^{b}(A)}\left(P_{j} \rightarrow P_{i}, P_{l} \rightarrow P_{t}\right)\right)= \\
= \begin{cases}0, & \text { if }\{i, i-1, \ldots, j\} \cap\{t, t-1, \ldots, l\}=\varnothing ; \\
0, & \text { if }\{i, i-1, \ldots, j\} \subset\{t, t-1, \ldots, l\}, i \neq t, j \neq l ; \\
1, & \text { if }\{i, i-1, \ldots, j\} \subset\{t, t-1, \ldots, l\}, i=t, j \neq l \\
1, & \text { if }\{i, i-1, \ldots, j\} \subset\{t, t-1, \ldots, l\}, i \neq t, j=l ; \\
2, & \text { if }\{i, i-1, \ldots, j\}=\{t, t-1, \ldots, l\} .\end{cases}
\end{gathered}
$$




$$
\begin{aligned}
& \operatorname{dim}\left(\operatorname{Hom}_{K^{b}(A)}\left(P_{m}, P_{j} \rightarrow P_{i}\right)\right)= \begin{cases}1, & \text { if } m=j ; \\
0, & \text { if } m \neq j .\end{cases} \\
& \operatorname{dim}\left(\operatorname{Hom}_{K^{b}(A)}\left(P_{j} \rightarrow P_{i}, P_{m}\right)\right)= \begin{cases}1, & \text { if } m=j ; \\
0, & \text { if } m \neq j .\end{cases} \\
& \operatorname{dim}\left(\operatorname{Hom}_{K^{b}(A)}\left(P_{m}, P_{r}\right)\right)= \begin{cases}k, & \text { for } m \neq r ; \\
k+1, & \text { otherwise. }\end{cases}
\end{aligned}
$$

These data give us the partition of the vertices of $\operatorname{End}_{K^{b}(A)}\left(T_{S}\right)$ into $A$-cycles or equally which edges of the Brauer tree of algebra $\operatorname{End}_{K^{b}(A)}\left(T_{S}\right)$ are incident to the same vertex (we will identify the edges of the Brauer tree of $\operatorname{End}_{K^{b}(A)}\left(T_{S}\right)$ and the indecomposable summand of $T_{S}$ corresponding to them). Now we have to find out the cyclic ordering of the edges incident to one vertex and which vertex is exceptional. Note that if we arrange the vertices of the $A$-cycle of length $r$ in such a manner that successive composition of $k r$ morphisms (in the case of the exceptional vertex) or of $r$ morphisms (in the case of a nonexceptional vertex) between them is not homotopic to zero then this arrangement will give us the desired cyclic order.

In the case when all stalk complexes of projective modules are concentrated in degree 0 in the algebra $\operatorname{End}_{K^{b}(A)}\left(T_{S}\right)$, the following types of $A$-cycles can occur: a) the $A$-cycle of projective modules; b) an $A$-cycle containing an indecomposable stalk complex of a projective module $P$ concentrated in degree 0 and two-term complexes having $P$ as a 0 -component; c) an $A$-cycle containing two-term complexes with the same 0-components; d) an $A$-cycle containing two-term complexes with the same components in degree 1.

For convenience let us use the following notation: a homomorphism $P_{l} \rightarrow P_{m}$ induced by multiplication on the right by $\alpha_{l} \alpha_{l+1} \ldots \alpha_{m-1}$ will be denoted by $\alpha_{l, m-1}$.

a) Let $\left(m_{1}\right),\left(m_{2}\right), \ldots,\left(m_{r}\right) \in S$ where the set $\left\{m_{1}, m_{2}, \ldots, m_{r}\right\}$ is ordered according to the cyclic ordering of the edges in the Brauer star, $r$ is maximal. It is clear that the following diagram of chain maps holds:

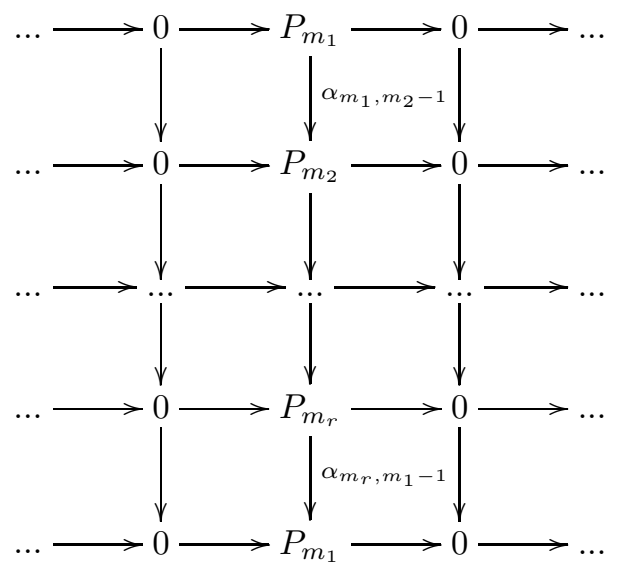


The successive composition of any $k r$ morphisms is not homotopic to 0 . So the edges of $\operatorname{End}_{K^{b}(A)}\left(T_{S}\right)$ corresponding to stalk complexes of projective modules have a common vertex and the cyclic ordering in $\operatorname{End}_{K^{b}(A)}\left(T_{S}\right)$ is induced by the cyclic ordering in the Brauer star. The vertex of $\operatorname{End}_{K^{b}(A)}\left(T_{S}\right)$ corresponding to this cycle is exceptional.

b) Let $\left(m_{1}, m_{1}-1, \ldots, j\right),\left(m_{2}, m_{2}-1, \ldots, j\right), \ldots,\left(m_{r}, m_{r}-1, \ldots, j\right),(j) \in S$, where the set $\left\{j, m_{1}, m_{2}, \ldots, m_{r}\right\}$ is ordered according to the cyclic ordering of the edges in the Brauer star, $r$ is maximal. Let us consider the following diagram of chain maps:

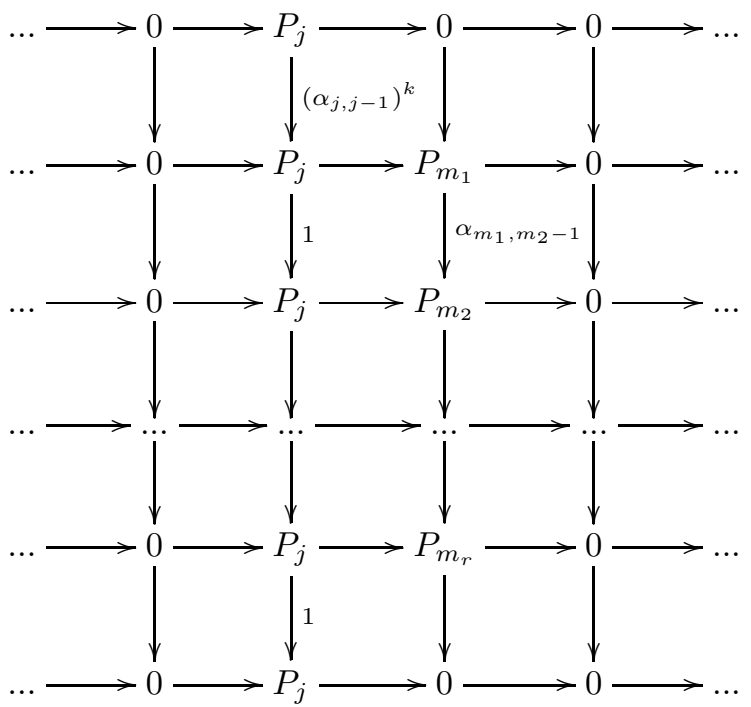

The successive composition of any $r+1$ morphisms is not homotopic to 0 . That means that the edges of $\operatorname{End}_{K^{b}(A)}\left(T_{S}\right)$ corresponding to this $A$-cycle are ordered in the following way: $\left\{P_{j}, P_{j} \rightarrow P_{m_{1}}, P_{j} \rightarrow P_{m_{2}}, \ldots, P_{j} \rightarrow P_{m_{r}}\right\}$.

c) Similarly, if $\left(m_{1}, m_{1}-1, \ldots, j\right),\left(m_{2}, m_{2}-1, \ldots, j\right), \ldots,\left(m_{r}, m_{r}-1, \ldots, j\right) \in S$ is the set of intervals corresponding to some $A$-cycle in $\operatorname{End}_{K^{b}(A)}\left(T_{S}\right)$, where the set $\left\{m_{1}, m_{2}, \ldots, m_{r}\right\}$ is ordered according to the cyclic ordering of the edges in the Brauer star, $r$ is maximal, then the edges of $\operatorname{End}_{K^{b}(A)}\left(T_{S}\right)$ corresponding to this $A$-cycle are ordered in the following way: $\left\{P_{j} \rightarrow P_{m_{1}}, P_{j} \rightarrow P_{m_{2}}, \ldots, P_{j} \rightarrow P_{m_{r}}\right\}$.

d)Let us now consider an $A$-cycle containing the summand with the same components in degree 1 . Let $\left(j, j-1, \ldots, m_{1}\right),\left(j, j-1, \ldots, m_{2}\right), \ldots,(j, j-$ $\left.1, \ldots, m_{r}\right) \in S$, where the set $\left\{m_{1}, m_{2}, \ldots, m_{r}\right\}$ is ordered according to the cyclic ordering of the edges in the Brauer star, $r$ is maximal. Then the following dia- 
gram of chain maps holds:

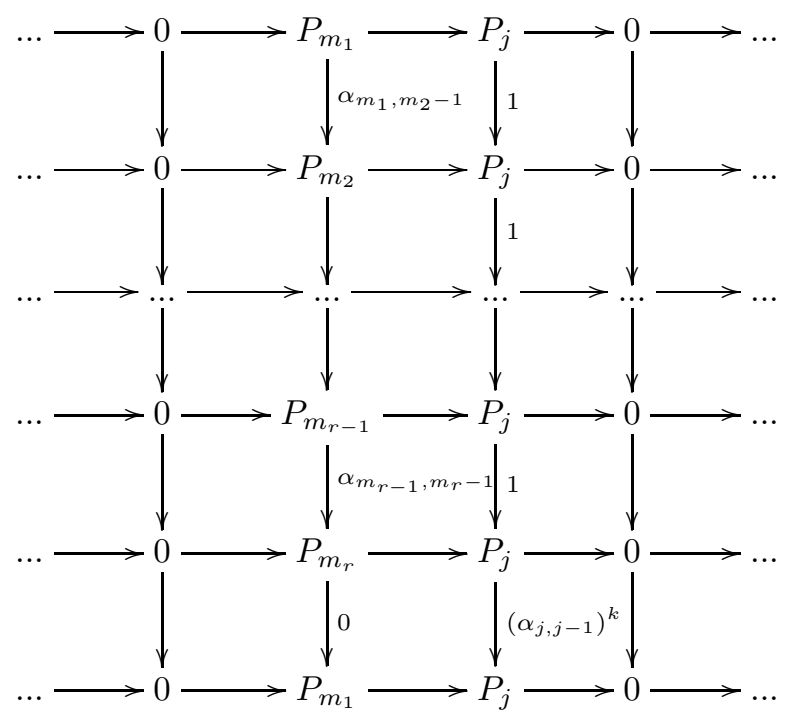

The successive composition of any $r$ morphisms is not homotopic to 0 . This means that the edges of $\operatorname{End}_{K^{b}(A)}\left(T_{S}\right)$ corresponding to this $A$-cycle are ordered in the following way: $\left\{P_{m_{1}} \rightarrow P_{j}, P_{m_{2}} \rightarrow P_{j}, \ldots, P_{m_{r}} \rightarrow P_{j}\right\}$.

This completes the first case. Since for each of the 4 cases of $A$-cycles we have described the cyclic ordering of vertices, which is naturally induced by the cyclic ordering of the vertices in the Brauer star algebra.

2) Let us consider the second case. To all outer intervals $(i, i-1, \ldots, j) \in S$ of length greater than 1 an inner interval $(i)$ of length 1 is assigned, i.e. all stalk complexes of projective modules which are direct summand of $T_{S}$ are concentrated in degree 1 . Let $(i, i-1, \ldots, j),(t, t-1, \ldots, l) \in S$ be two arbitrary intervals of length greater than 1 . And let $(m),(r) \in S$ be intervals of length 1 . It is easy to see that

$$
\begin{aligned}
& \operatorname{dim}\left(\operatorname{Hom}_{K^{b}(A)}\left(P_{j} \rightarrow P_{i}, P_{l} \rightarrow P_{t}\right)\right)= \\
& = \begin{cases}0, & \text { if }\{i, i-1, \ldots, j\} \cap\{t, t-1, \ldots, l\}=\varnothing ; \\
0, & \text { if }\{i, i-1, \ldots, j\} \subset\{t, t-1, \ldots, l\}, i \neq t, j \neq l ; \\
1, & \text { if }\{i, i-1, \ldots, j\} \subset\{t, t-1, \ldots, l\}, i=t, j \neq l \\
1, & \text { if }\{i, i-1, \ldots, j\} \subset\{t, t-1, \ldots, l\}, i \neq t, j=l ; \\
2, & \text { if }\{i, i-1, \ldots, j\}=\{t, t-1, \ldots, l\} .\end{cases} \\
& \operatorname{dim}\left(\operatorname{Hom}_{K^{b}(A)}\left(P_{m}, P_{j} \rightarrow P_{i}\right)\right)= \begin{cases}1, & \text { if } m=i \\
0, & \text { if } m \neq i .\end{cases} \\
& \operatorname{dim}\left(\operatorname{Hom}_{K^{b}(A)}\left(P_{j} \rightarrow P_{i}, P_{m}\right)\right)= \begin{cases}1, & \text { if } m=i \\
0, & \text { if } m \neq i\end{cases} \\
& \operatorname{dim}\left(\operatorname{Hom}_{K^{b}(A)}\left(P_{m}, P_{r}\right)\right)=\left\{\begin{array}{l}
k, \text { for } m \neq r \\
k+1, \text { otherwise. }
\end{array}\right.
\end{aligned}
$$


As in the previous case, the exceptional vertex corresponds to the cycle of stalk complexes of projective modules (this time they are concentrated in degree 1). All $A$-cycles can be divided into 4 types. For 3 of them (namely a, c, d) we already know the cyclic ordering. The remaining case is:

e) Let $\left(j, j-1, \ldots, m_{1}\right),\left(j, j-1, \ldots, m_{2}\right), \ldots,\left(j, j-1, \ldots, m_{r}\right),(j) \in S$, where the set $\left\{j, m_{1}, m_{2}, \ldots, m_{r}\right\}$ is ordered according to the cyclic ordering of the edges in the Brauer star, $r$ is maximal. Let us consider the following diagram of chain maps:

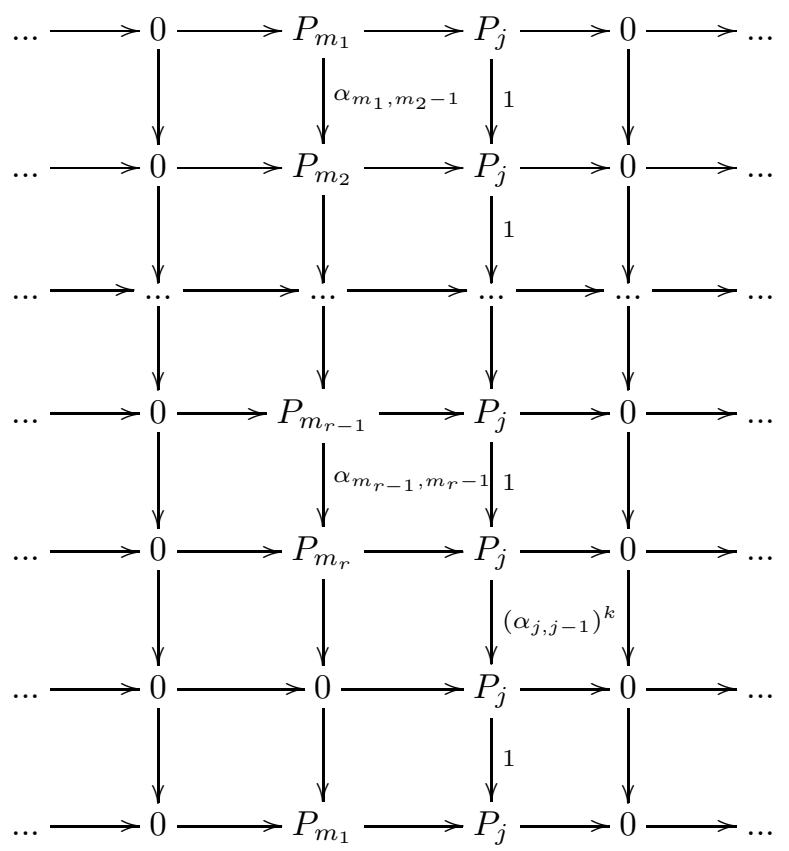

The successive composition of any $r+1$ morphisms is not homotopic to 0 . This means that the edges of $\operatorname{End}_{K^{b}(A)}\left(T_{S}\right)$ corresponding to this $A$-cycle are ordered in the following way: $\left\{P_{m_{1}} \rightarrow P_{j}, P_{m_{2}} \rightarrow P_{j}, \ldots, P_{m_{r}} \rightarrow P_{j}, P_{j}\right\}$.

The following is clear from the description of endomorphism rings of twoterm tilting complexes.

Remark 2. A two-term tilting complex $T_{S}$ over a Brauer star algebra with $n$ edges and multiplicity 1 , which is not isomorphic to $A$ or $A[-1]$, gives a derived autoequivalence if and only if the covering $S$ of the n-gon has the following form:

$$
(j, j-1, \ldots, j+1),(j, j-1, \ldots, j+2), \ldots,(j, j-1),(j) j=1, \ldots, n
$$

or

$$
(j-1, j-2, \ldots, j),(j-2, j-3, \ldots, j), \ldots,(j+1, j),(j), j=1, \ldots, n .
$$

The subgroup of the derived Picard group generated by these autoeqivalences was studied in [2]. 
In the case $k \neq 1$ a two-term tilting complex $T_{S}$ gives a derived autoequivalence if and only if the covering $S$ is trivial.

Let us consider an example of a two-term tilting complex and compute its endomorphism ring.

Example 1. Let $k=1, n=4$. And let $S=(1,2,3,4),(2,3,4),(2,3),(1)$.

Then $T_{S}$ consists of the following direct summand: $P_{4} \rightarrow P_{1}, P_{4} \rightarrow P_{2}, P_{3} \rightarrow$ $P_{2}$ and $P_{1}$, concentrated in degree 1 . Let us denote the vertices of $\operatorname{End}_{K^{b}(A)}\left(T_{S}\right)$ as follows: $a$ is a vertex corresponding to $P_{4} \rightarrow P_{1}, b$ to $P_{4} \rightarrow P_{2}, c$ to $P_{3} \rightarrow P_{2}$, $d$ to $P_{1}$. Then the quiver of $\operatorname{End}_{K^{b}(A)}\left(T_{S}\right)$ is of the following form:

$$
d \leftrightarrows a \leftrightarrows b \leftrightarrows c
$$

and the Brauer graph is a string:

Proposition 5. For any algebra $B$ corresponding to the Brauer tree $\Gamma$ with $n$ edges and multiplicity $k$ there is a two-term tilting complex $T_{S}$ over $A$ such that $B \simeq \operatorname{End}_{K^{b}(A)}\left(T_{S}\right)$.

Proof Let us assume that the root of $\Gamma$ is chosen in the exceptional vertex, and that $\Gamma$ is embedded in the plane in such a manner that all nonroot vertices are situated on the plane lower than the root according to their level (the further from the root, the lower, all vertices of the same level lie on a horizontal line). The edges around vertices are ordered clockwise.

Let us number the edges of the tree $\Gamma$ as follows: put 1 on the right-hand edge incident to the root, on the next edge incident to the root according to the order put $1+k_{1}+1$, where $k_{1}$ is the number of successors of the nonroot end of the edge with label 1 . Let the $(i-1)$-st edge incident to the root be labelled with $m$ and let the nonroot vertex incident to the edge with label $m$ have $k_{m}$ successors, then put on the $i$-th edge incident to the root label $m+k_{m}+1$.

Further on let us put the labels as follows: consider a vertex of an odd level (a vertex which can be connected to the root by a path of odd length), let the edge connecting it to the vertex of a higher level be labelled with $j$. Put $j+1+k_{1}$ on the right-hand edge incident to this vertex, where $k_{1}$ is the number of successors of the other end of this edge. Put $j+1+k_{1}+k_{2}+1$ on the next edge incident to this vertex, where $k_{2}$ is the number of successors of the other end of this edge. Further on let us put the labels by induction: let the $(i-1)$-st edge incident to the fixed vertex be labelled with $m$, and let the lower end of the next edge have $k_{m}$ successors, put $m+k_{m}+1$ on the $i$-th edge incident to this vertex.

Consider a vertex of an even level, let the edge connecting it to the vertex of a higher level be labelled with $t$ and let the edge connecting the other end of the edge labelled with $t$ and the vertex of a higher level be labelled with $j$. Put $j+1$ on the right-hand edge incident to this vertex. Put $j+1+k_{j+1}+1$ on the next edge incident to this vertex, where $k_{j+1}$ is the number of successors of the other end of the edge labelled with $j+1$. Let the $(i-1)$-st edge incident to the fixed vertex be labelled with $m$, and let the lower end of the $(i-1)$-st edge 
incident to the fixed vertex have $k_{m}$ successors, put $m+k_{m}+1$ on the $i$-th edge incident to this vertex.

Let us construct a tilting complex over algebra $A$ using a labelled tree $\Gamma$. Assume that the root of $\Gamma$ has $l$ children and there are labels $\left\{n_{1}, n_{2}, \ldots, n_{l}\right\}$ on the edges incident to the root. Take stalk complexes of projective modules $P_{n_{1}}, P_{n_{2}} \ldots, P_{n_{l}}$ concentrated in degree 0 as summands of the tilting complex. Let us consider a vertex of an odd level. Assume that the edge connecting it to a vertex of a higher level is labelled by $j$, the other edges incident to this vertex have labels $j_{1}, j_{2}, \ldots j_{h}$, where $h$ is the number of children of this vertex. In the tilting complex the following direct summands will correspond to these edges: $P_{j} \rightarrow P_{j_{1}}, P_{j} \rightarrow P_{j_{2}}, \ldots, P_{j} \rightarrow P_{j_{h}}$.

Let us consider a vertex of an even level. Assume that the edge connecting it to a vertex of a higher level is labelled by $g$, the other edges incident to this vertex have labels $g_{1}, g_{2}, \ldots, g_{d}$, where $d$ is the number of children of this vertex. In the tilting complex the following direct summands will correspond to these edges: $P_{g_{1}} \rightarrow P_{g}, P_{g_{2}} \rightarrow P_{g}, \ldots, P_{g_{d}} \rightarrow P_{g}$. It is clear that we have the desired number of summands. Because of the construction this complex is tilting and the Brauer tree corresponding to its endomorphism ring is $\Gamma$.

Similarly, we could construct a tilting complex with all the stalk complexes of projective modules concentrated in degree 1.

\section{References}

[1] R. Rouquier, A. Zimmermann, Picard groups for derived module categories, Proc. London Math. Soc. (3) 87 (2003), no. 1, 197-225.

[2] I. Muchtadi-Alamsyah, Braid action on derived category of Nakayama algebras, Comm. Algebra 36 (2008), no. 7, 2544-2569.

[3] H. Abe, M. Hoshino, On derived equivalences for selfinjective algebras, Comm. in Algebra 34 (2006), no. 12, 4441-4452.

[4] M. Schaps, E. Zakay-Illouz, Combinatorial partial tilting complexes for the Brauer star algebras, Proc. Int. Conference on Representations of Algebra, Sao Paulo, (2001), 187-208.

[5] M. Schaps, E. Zakay-Illouz, Pointed Brauer trees, J. Algebra, 246 (2001), no. $2,647-672$.

[6] J. Rickard, Morita theory for derived categories, J. London Math. Soc. 39 (1989), no. 2, 436-456.

[7] I.M. Gelfand, V.A. Ponomarev, Indecomposable representations of the Lorentz group, Russian Math. Surveys 23 (1968), no. 2(140), 3-59.

[8] B. Wald, J. Waschbüsch, Tame biserial algebras, J. Algebra 95 (1985), 480500 . 
[9] M. A. Antipov, A. I. Generalov, Finite generation of the Yoneda algebra of a symmetric special biserial algebra, Algebra i Analiz, 17:3 (2005), 123.

[10] J. L. Alperin, Local representation theory, Cambridge studies in advanced mathematics 11, Cambridge University Press (1986).

[11] J. Rickard, Derived categories and stable equivalence, J. Pure Appl. Algebra, 61 (1989), 303-317.

[12] P. Gabriel, C. Riedtmann, Group representations without groups, Comment. Math. Helv. 54 (1979), 240-287.

[13] M. C. R. Butler, C. M. Ringel, Auslander-Reiten sequences with few middle terms and applications to string algebras, Comm. Algebra, 15 (1-2) (1987), $145-179$.

[14] D. Happel, Auslander-Reiten triangles in derived categories of finitedimensional algebras, Proc. Amer. Math. Soc. 112 (1991), 641-648.

[15] D. Happel, Triangulated Categories in the Representation of Finite Dimensional Algebras, Cambridge University Press (1988). 